\title{
The Role of Hyperspectral Imaging: A Literature Review
}

\author{
Muhammad Mateen, Junhao Wen, Nasrullah, Muhammad Azeem Akbar \\ School of Big Data and Software Engineering, \\ Chongqing University, \\ Chongqing, 401331, China
}

\begin{abstract}
Optical analysis techniques are used recently to detect and identify the objects from a large scale of images. Hyperspectral imaging technique is also one of them. Vision of human eye is based on three basic color (red, green and blue) bands, but spectral imaging divides the vision into many more bands. Hyperspectral remote sensors achieve imagery data in the form of hundreds of adjoining spectral bands. In this paper, our purpose is to illustrate the fundamental concept, hyperspectral remote sensing, remotely sensed information, methods for hyperspectral imaging and applications based on hyperspectral imaging. Moreover, in the forensic context, the novel methods involving deep neural networks are elaborated in this paper. The proposed idea can be useful for further research in the field of hyperspectral imaging using deep learning.
\end{abstract}

Keywords-Deep learning; electromagnetic spectrum; hyperspectral imaging; imaging spectroscopy; multispectral imaging; remote sensing

\section{INTRODUCTION}

In hyperspectral, the term "hyper" means "too many" and it refers to the huge amount of measured wavelength bands. Hyperspectral images are used to provide sufficient spectral information to recognize and differentiate spectrally distinctive materials. Optical analysis techniques are used to detect and identify the objects from a large scale of images. Hyperspectral imaging technique is one of them. Vision of human eye is based on three basic colors (red, green and blue) bands, but spectral imaging divides the vision into many more bands. Hyperspectral remote sensors achieve imagery data in the form of hundreds of adjoining spectral bands.

The collective data is used to obtain a constant spectrum for each imagery pixel mentioned in Fig. 1. After tuning the sensor, terrain and atmospheric belongings are applied [1]. These imagery spectrums can be analyzed with laboratory or field reflectance spectra to know and map surface materials such as specific kind of plants or indicative minerals with ore deposits.

Imaging spectrometers are instruments used to produce hyperspectral images. The remote imaging and spectroscopy are two basic technologies used to develop the hyperspectral sensors. Spectroscopy is a field of study about light emission or reflection from different materials and the changes occurred in energy with wavelength.

In the field of optical remote sensing, spectroscopy acts with the spectra of sunlight that is scattered by objects at or inside the earth. Remote images are planned to capture and calculate the light returned from adjoining areas on the surface of earth. Hyperspectral imaging can be applied to various applications including medicines, biogeochemistry, biophysics, industrial monitoring and remote sensing to collect the information for analysis.

Chinese Academy of Science played a vital role in the field of hyperspectral imaging and developed two outstanding imagers, one of them is known as Push broom Hyperspectral Imager (PHI) and another one is Operative Modular Imaging Spectrometer (OMIS). In 2000, another invention about imaging came out in the form of Hyperspectral Digital Camera (HSDC)which supports limited spectral bands with high quality of spectral resolution [2]. HSDC plays a flexible role for different observation objects and applications including environmental and agricultural monitoring.

Analysis of Hyperspectral imager can be performed by two different ways one of them is perspective of spectral analysis and another is based on image processing. It is more important that the data should be well organized before selection of any kind of approach. In spectroscopic analysis, the spectra should be extracted by region of interest that is usually calculated by three different ways such as threshold an image with single waveband, ratio or difference image. In image processing, few images are selected from the collection of images for rapid computation. Selection of those images is based on the importance of their wavelength for shifting carefully. In Fig. 2 [3] for careful shifting, spectra provides an option for peaks and valleys whether in [4] based on original or preprocessed format.

There are several other methods to achieve the same goal, for example, partial least square regression and principal component analysis. Additionally, some data compression techniques such as singular value decomposition and Fourier transform are used for the process of more images to increase the ability of hyperspectral imaging [5]. After the achievement of healthy data, the next step is to make dependent standardized models. Before implementation of Chemometric algorithm, it is compulsory to overcome the noisy data to increase the quality of signals. Moreover, image processing includes filtering and binning, which can increase the quality of data. In calibration model, the same routine is followed by spectroscopy and spectral analysis as shown in Fig. 2. 


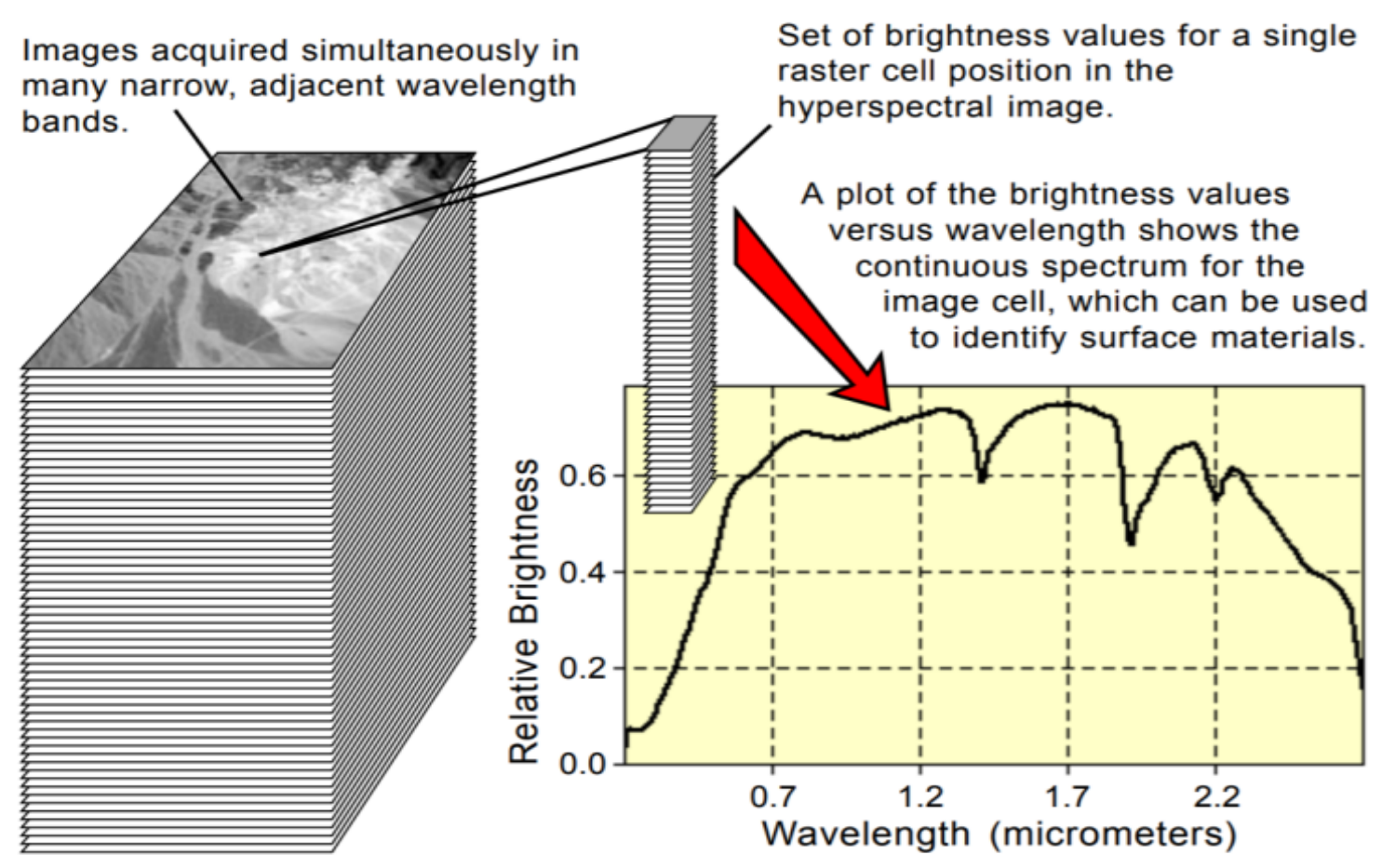

Fig. 1. Measurement of hyperspectral remote sensor.

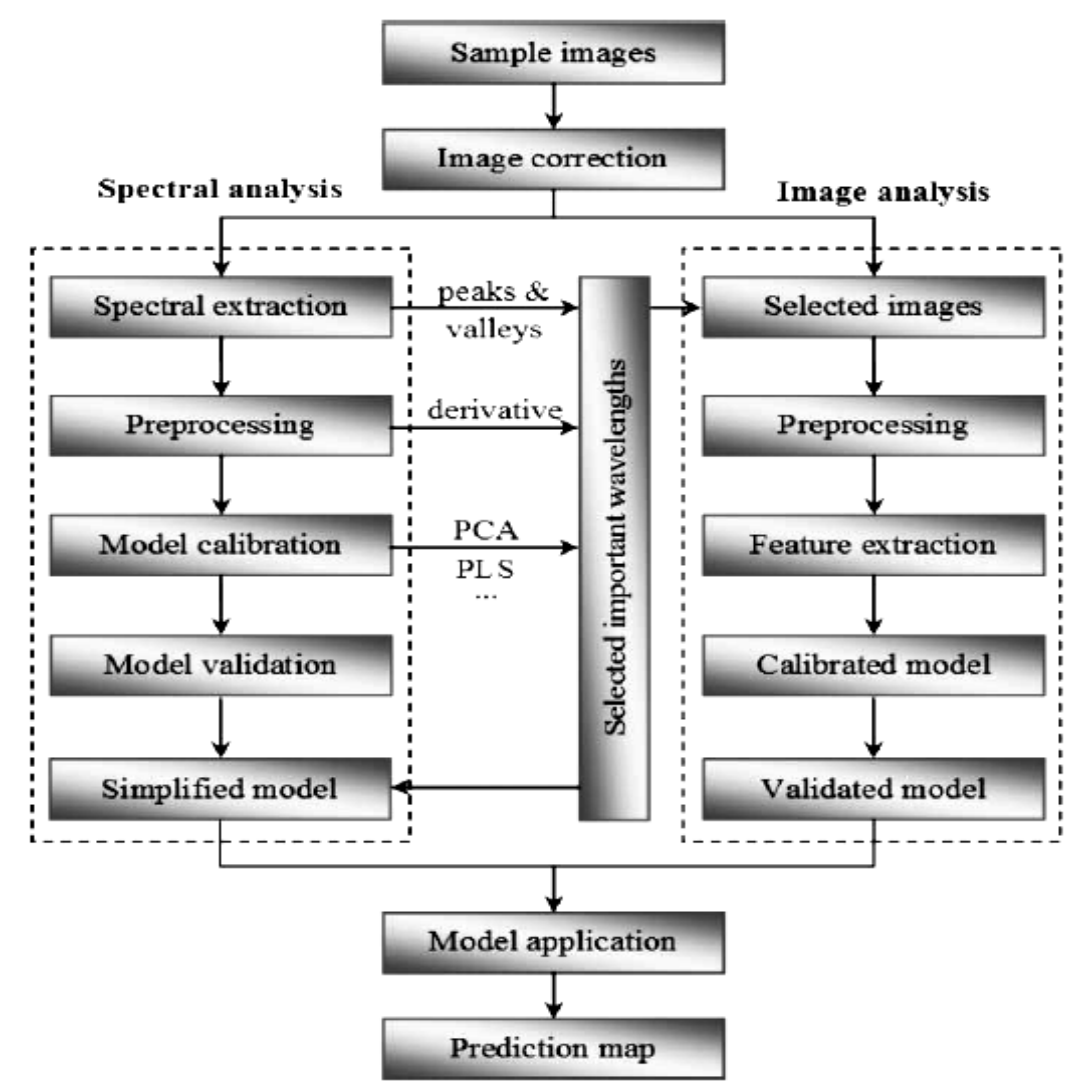

Fig. 2. Flowchart of hyperspectral imaging processing.

In this model different techniques such as principal component analysis, partial least square discriminate analysis, fisher's linear discriminate technique, support vector machine, artificial neural network and fuzzy inference are offered [3]. As a result prediction map can be progressed with the use of post- processing techniques and the information is achieved. 
The rest of the paper is organized as: related work of the study is discussed in Section II. Study findings are reported in Section III, and Section IV covers the future work of the study. The applications of hyperspectral imaging are explained in Section V. Finally, the findings are concluded in Section VI.

\section{RELATED WORK}

Light emitted by sun strikes on the molecules, causing in absorption and reflection of light which depends on the structure or architecture of molecules. The wavelength of absorption or reflection of atomic bonds and molecule makes detection or identification of a specific object. To collect the amount of light reflected by a specific object or earth surface is used for identification of that object. There are different types of sensors used to collect the scattered data including truck-mounted sensor, airborne sensor and satellite-based sensor. With the help of fast computers and development of sensors, the immense research [6] is being carried out in the field of "Hyperspectral remote sensing".

In [7], Sun, L et al. introduced a novel approach based on supervised classification for hyperspectral imaging. Supervised classification model includes a spatially weighted random fields and spectral data reliability. To progress the quality of classification, the real labels of training data samples are fixed as a constraint in the model of proposed approach. In [8], Sun, L. et al. introduced noise (deadlines, strip noises, impulse and Gaussian) reduction technique for hyperspectral imaging with the use of low-rank representation on the spectral image. According to the proposed technique spectral space of hyperspectral image recline in the low-rank subspace. The low rank representation based on spectral space was oppressed by nuclear norm of image with the spectral dimension. It causes potential for the removal of deadlines, strips, sparse noise and Gaussian noise on the level of each band.

In the field of image classification, high dimensionality of imaging data is a challenge. Hyperspectral imaging data also contains high dimensionality, so the spatial-spectral classification methods are demands for computational view point. In [9], $\mathrm{Wu}, \mathrm{Z}$. et al. introduced a novel technique for parallel implementation of spatial-spectral classification on the basis of adaptive random fields. In this technique, logistic regression classifier is used for spectral information. In this research, graphics processing units are used for parallel implementation. GPU sets the work allocation, input and output between the CPU and the graphics processing unit having fully computational control of GPUs with low latency and high bandwidth of shared memory.

In [10], Sun, L. et al. introduced a novel approach for noise reduction in the hyperspectral images. Noise reduction technique is based on the super pixel-based low level rank of representation for hyperspectral imaging. Under the model of a linear mixture, the hyperspectral cube is considered as a low rank in the spectral area, which is divided HSI data into the sub-matrices of lower ranks.

In 1985, a technique named as "Imaging spectrometry" was used for the remote sensing of the earth. In addition, there was a motivation for the development of digital image processing methodologies by multispectral data analysis [11]. In the early stage, Goetz et al. [6] defined Hyperspectral remote sensing as "The collection of images in dozens to hundreds of adjacent registered spectral bands so that for every cell of an image a glowing spectrum can be calculated." According to this definition, spectral regions of VIS (visible), NIR (Near Infrared), SWIR (Shortwave Infrared), MWIR (Midwave Infrared), LWIR (Longwave Infrared) and UV (Ultraviolet) are covered.

\section{A. Hyperspectral Remote Sensing}

The hyperspectral sensor is used to study soil science, geology, mining, land use, and hydrology to map and identify geometric and chemical patterns of land. Information obtained by hyperspectral sensor is used to identify valuable minerals and petroleum. Imaging spectroscopy is a technique used to absorb specific features of chemical bonds in minerals. Material mapping includes water, ice, snow, mineral mixtures, vegetation, environmental materials, atmospheric gases, and man-made materials. Hyperspectral airborne scanners were used in 1998 to identify rocks and soils containing hydrocarbon [12]. This experiment was named as "Pro Smart Experiment" managed by German Aerospace Center to test the Hyperspectral "HyMap" designed by the Australian Company Integrated Spectronics Ltd. In Hyperspectral data analysis, several factors are involved to make it complex for refined techniques and algorithms.

To demonstrate the hyperspectral data analysis, themes are managed in six main areas including un-mixing, data fusion, target detection, physical parameter retrieval, classification and fast computing based on image processing, machine learning, and signal processing. According to [13], researchers described all the areas one by one in the paper. In the data fusion, various strategies are defined including restoration of the signal to noise ratio (SNR) and spatialresolution, spectral data fusion to overcome high spectral redundancy, spatial data fusion used to enhance the resolution of imaging sensor at the sub-pixel level. There is another sensor named as "Dual push broom hyperspectral sensor" which contains two various devices (covers different wavelengths) that are normally attached to the similar bench. "Co-registration of pixels" and "Peer of the field of view" are problems lead by this configuration called Bore sight Effect. To observe and detect spectral phenomena, A. Brook et al. [14] proposed a prior to its correction with the use of Bore sight Effect that is used to provide supplementary information not located in the corrected images.

\section{B. Remotely Sensed Data}

Hyperspectral sensors collect the data from the surface of the earth with the help of different parameters. It is important to measure the accurate values of identified objects. There are a lot of methods and techniques, developed to measure the values of vegetation. Some techniques show that the results can be obtained in highly accurate form but few techniques show the results in moderately accurate form.

In [15], Mutanga et al. studied on a small number of chemicals, for example, chlorophyll and another kind of pigments. The pigments including carotenes and chlorophyll 
are identifiable with 80 per cent accuracy in sample fields. Nitrogen can also be detected with accuracy. But the other nutrients namely magnesium, calcium, sodium, potassium, and phosphorus have less notified by Mutanga et al. in his research. Even though this is very expensive for projects to analyze with a lot of samples, the impending of remote sensing is not totally oppressed. In the [16], research related with Geoinformation science and earth analysis for modeling and management of environment designed a remote sensing data model in which "spot vegetation" concerned with georeferenced and "Corine land cover data" concerned with study area that provides qualitative and quantitative information of earth surface. This research work used datasets with some characteristics including geometric accuracy better than $100 \mathrm{~m}$, thematic accuracy greater than 85 percent, and spatial resolution of $30 \mathrm{~m}$.

For the development of South African Imaging Spectroscopy Landscape, an overview is explained in brief about analysis techniques and challenges [17]. According to African research and application, indicators can be derived by the full width of the spectral response for example slopes, integrals, and curve derivatives. The purpose is to identify data source from the imaging spectroscopy, in which spectral attributes are required to notify an application of interest. According to [18], object-based classification approach is used to classify the remotely sensed data. In 2000, Walter and Fritsch introduced a concept using multispectral remote sensing data to automatically revise the geographical information system databases. In object-based classification, not only single pixel is classified but the group of pixels is also classified based on geographical information system database. This approach depends on two main steps, the first step is based on supervised likelihood classification and the second step is based on matching of existing GIS objects with classified objects to detect the change occurred or to identify the faulty data. This approach develops and makes the better result of the classification. At the end, the result is obtained in the form of fully classified, partly classified and not found by the use of a threshold, iteratively defined by a user. In [19], a surface bidirectional reflectance model has been designed. The model is applicable for heterogeneous surfaces and follows a semi-empirical approach. There are three parameters used to describe the nature of the surface and these parameters are used in the effective algorithm of correction and processing the remote sensing data. The model based on surface bidirectional reflectance is collection of two basic processes, one of them is to diffuse reflection component which explains the geometrical structure on surface and also understands the shadow effects while the second process defines the volume scattering properties obtained by isolated surfaces. Invisible and near-infrared spectral bands, model and situ annotations demonstrate the better association for common investigated surface types. The model is good to reduce the largely unwanted variations related to surface bidirectional results in remotely sensed data.

U.C. Benz et al. [20] proposed object-oriented analysis associated with fuzzy methods to implement information and explained workflow from remote sensing imagery to graphical information system. During proposed method, software named as "eCognition" is used to offer the suitable connection between remote sensing imagery and graphical information system. Thus object-oriented technique can provide powerful automatic and semiautomatic evaluation to allocate practiced knowledge to GIS.

\section{DISCUSSIONS}

Hyperspectral imagery process follows some methods to accomplish the proposed task. These methods contain study area, which means where experiment is performed; field sampling, meaning the sample data gathered from study area; Chemical analysis, which means evaluation of chemical compounds and Spectral processing, which means to perform operation on sample of spectra to overcome noisy data, statistical formulas are used for prediction of parameters. D. Liao et al. [21] proposed method to visualize hyperspectral image in normal color by the coordination of HSI and high resolution image through multiple alignments. Manifold alignment identifies the matching color points and displays it into pair wise alignment, while the spaces between them play the role of bridges. Association of hyperspectral imaging and RGB image generates a spatial image in the natural format. This approach has advantage of flexibility that's why it can be applied for different scenario. The same approach is also well known because of user's customization, in which user can scan visual resultant bands according to the specified interest. Chemometric and spectroscopy areas provided methods and analysis tools including "Partial Least Squares Regression Analysis" and "Principal Component", which are useful for the process of hyperspectral image [22]. Workflow of hyperspectral image processing is different from the workflow of color image processing but both data types are multivariate and multidimensional. Hyperspectral imaging methods such as image acquisition, spectral and spatial preprocessing, dimensionality reduction, calibration, feature extraction and selection are used for HSI processing as shown in Fig. 2. Push broom line scanner is commonly used as distributed hyperspectral imaging sensor. Push broom scans all wavelength data points with the same spatial coordinates. In the hyperspectral imaging system, calibration is a method that ensures the repeatability and accuracy of results gained by hyperspectral imaging data. Calibration is a procedure that connects wavelengths with band numbers. Spatial calibration is a method of measuring the correlation of each image pixel such as meters. Spatial preprocessing is also a method used in hyperspectral imaging to minimize the noisy data from the images. Spatial post-processing is considered more valuable than spatial preprocessing because prediction and classification images are common images that need spatial manipulation, interpretation and pattern identification. Spatial sampling and Region of Interest (ROI) is a basic method of hyperspectral imaging used to mention the study area or location of interest for hyperspectral sensors. Spatial sampling can also be considered as background masking produced by binary images [22]. Transformation to absorbance is also used during reflectance or transmittance of data for analysis [23]. Hyperspectral imaging contains noisy data during scanning a large scale of image. To reduce noisy data, de-noising algorithm is performed in the spectral domain of hyperspectral imaging [24]. Feature extraction is also essential method used 
to transform the existing features to a set of new features Selection of subset from input feature without transformation is called selection extraction. It is also called feature subset selection. Mosaic based images [25] which are merged into a particular hyperspectral image, is used for the data analysis and classification algorithms.

In [26], affinity propagation method is used for the selection of bands in hyperspectral imaging. In this method centered based clustering approach is used to classify the similar color bands. AP is applied by factor graph and then operation performed by the centralized data points through message passing until an appropriate set of bands is achieved. The presentation of band selection is analyzed by the classification of a pixel inside the specific image. Classification of pixels is better performed by affinity propagation instead of all other approaches. Unlike conventional methods of clustering named as K-means, agglomerative clustering, a proposed technique (AP) obtained better results of band selection through message passing method. Hyperspectral imaging contains massive information of colored bands. Collection of colored bands can have noisy data that is considered to remove before processing. During preprocessing manual band removal technique is used to remove the noisy data. In [27], problem related with MBR is identified, because during MBR an important data can also be removed. For this problem, a proposed technique is introduced to automatically select the noisy bands instead of MBR selection. In the proposed technique, first wavelet reduction is applied to de-noise the bands of specified image and then Affinity Propagation approach is used to classify the representative bands from the noisy data with a smart way. To overcome noisy data in an efficient way, two sensors are applied, so the experimental results show that proposed technique is better performed than Manual Band Removal technique.

To overcome the noisy data from hyperspectral images is also introduced by [28], a proposed technique of sparse representation based on noise reduction method. This technique depends on a non-noisy component which can be sparsely decayed over redundant dictionary rather than a noisy component. The paper shows the correlation of spectral-spatial structure of HSI by use of three dimensional blocks instead of two dimensional patches for sparse representation. Gaussian and Poisson noise models are collectively used for signaldependent and signal independent noises in hyperspectral imagery. The proposed technique is good for virtual and real data sets of HS remote sensing.

Naganathan et al. [29] proposed meat tenderness for the satisfaction of consumers. Purpose of this proposal was to build up and test a near infrared/visible hyperspectral imaging system to guess tenderness of cooked beef by hyperspectral images. For this purpose, a push-broom hyperspectral imaging system associated with diffuse floodlighting system was designed and standardized. Three tenderness categories including tender, intermediate and tough were used to identify the features of meat tenderness. Statistical textual features obtained from Slice Shear Force (SSF) analysis are used in "canonical discriminate model" for prediction. The results signify that hyperspectral imaging played a vital role in the prediction of meat tenderness.

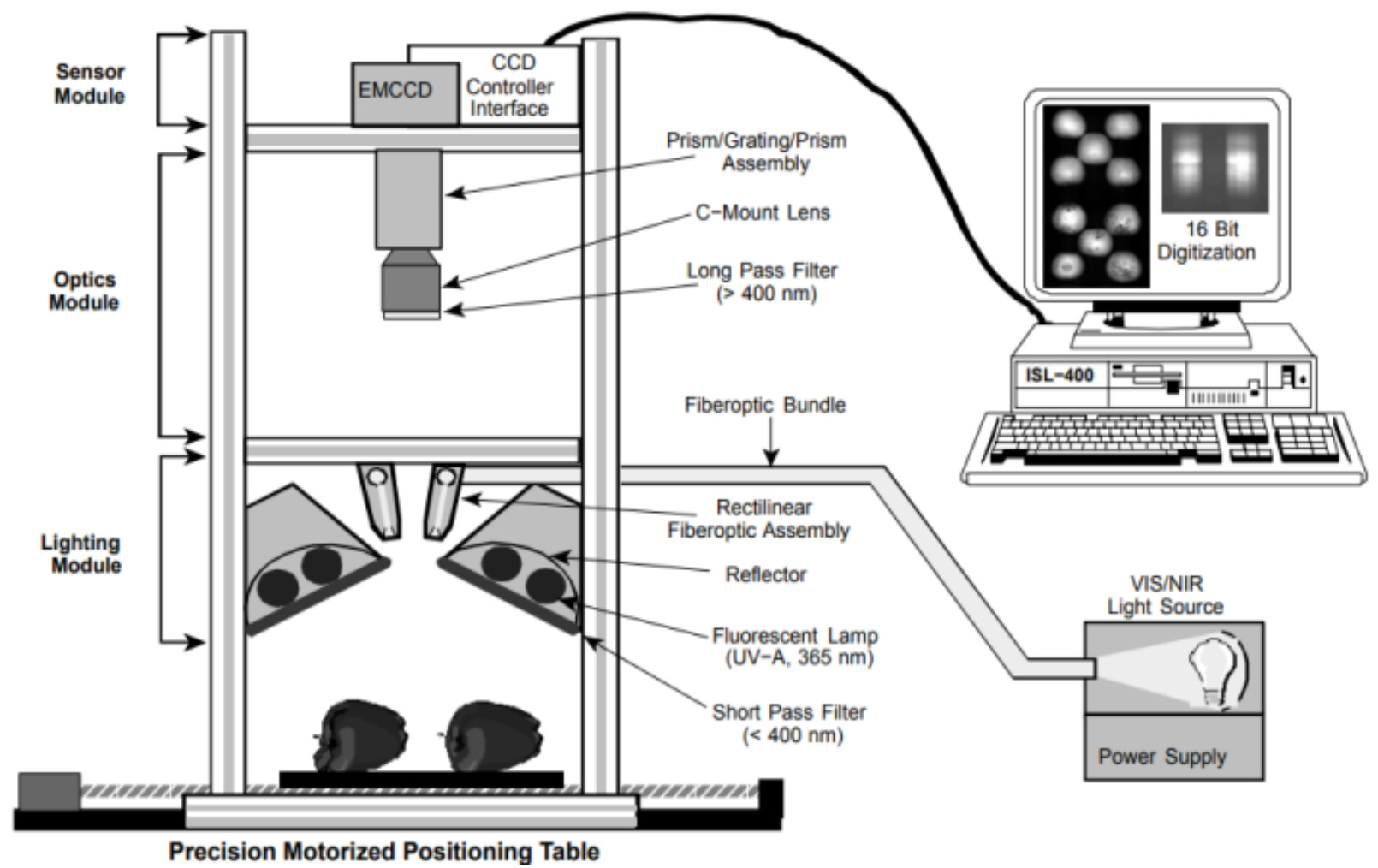

Fig. 3. Schematic diagram of the ISL hyperspectral imaging system. 
TABLE I. DETECTION RESUlts OF SKIN TUMOR

\begin{tabular}{|l|l|l|l|l|}
\hline Image & $\begin{array}{l}\text { No. of } \\
\text { Real } \\
\text { Tumors }\end{array}$ & $\begin{array}{l}\text { No. of } \\
\text { Detections }\end{array}$ & $\begin{array}{l}\text { No. of False } \\
\text { Positives }\end{array}$ & No. of Missing \\
\hline 1 & 3 & 3 & 1 & 0 \\
\hline 2 & 3 & 3 & 0 & 0 \\
\hline 3 & 1 & 1 & 1 & 0 \\
\hline 4 & 3 & 2 & 1 & 1 \\
\hline 5 & 4 & 4 & 1 & 0 \\
\hline 6 & 2 & 1 & 1 & 1 \\
\hline $7(2)$ & 3 & 3 & 1 & 0 \\
\hline $8(2)$ & 7 & 5 & 3 & 2 \\
\hline $9(2)$ & 3 & 3 & 3 & 0 \\
\hline 10 & 12 & 6 & 0 & 6 \\
\hline Total & $\mathbf{4 1}$ & $\mathbf{3 1}(\mathbf{7 6 \%})$ & $\mathbf{1 2}(\mathbf{2 8 \%})$ & $\mathbf{1 0}(\mathbf{2 4 \%})$ \\
\hline
\end{tabular}

Hyperspectral Imaging system is used for detection of skin tumor [30], in which first of all the carcass of chicken is analyzed for the usage of spectral information as shown in Fig. 3, and results are applied for the detection of skin tumor as shown in Table I.

There was a little deficiency about detection that tumor less than 3 millimeters in diameter could not be identified. This step in research opens a gate for the detection of tumors by hyperspectral imaging and can also be improved for computational complexity.

In the field of poultry farms, hyperspectral imaging is used to design a rapid, accurate and non-distractive method to detect the embryo and fertility improvement in eggs [31]. A near infrared spectral imaging system was introduced for the identification of fertility and early embryo improvement. For the experiment, totally 174 white shell eggs were used including 18 infertile eggs and 156 fertile eggs incubated for 4 days. During the inspection by hyperspectral imaging, eggs were categorized into two parts one for fertile and other for infertile and the dataset of each category is different with the day of incubation. Gabor filter used to extract the image texture information of eggs. $\mathrm{K}$ means clustering technique is also applied to cluster out the data and top results were achieved as $84.1 \%$ at day $4,81.8 \%$ at day $3,74.1 \%$ at day 2 and $100 \%$ at day 0 . The result shows that last three bands were used for detection because of maximum response of spectral transmission and can be applied for real-time detection system for early embryo and fertility of chicken eggs. In the field of hyperspectral imaging, a large number of researchers are focused to design different kinds of spectralspatial techniques while remaining are paying attention towards experimental development [32]. To evaluate the hyperspectral imaging classification techniques, it needs the reliable plan of testing including suitable standardized data sets, sampling procedure for training and testing data, and fair analysis standard [33]. J. Liang et al. [34], proposed random sampling approach for spatial-spectral techniques to decrease the overlapping between testing and training samples and offer more intention and precise evaluation. Random sampling approach [35] is often used because it cares all tagged data evenly and every sample is picked with the similar likelihood.

In [36], there is a review of hyperspectral imaging analysis with different techniques. In [36], Artificial Neural Network, Auto-Encoder, Stack Auto-Encoder, Conventional Neural Network and deep leaning is discussed for the analysis of Hyperspectral Imaging. After comparison of all these techniques and getting results, the author mentioned that deep learning outperformed in the analysis of hyperspectral imaging among all other stated techniques.

Furthermore in [37], there is a new model named as RVCANet designed in the context of deep learning for classification of hyperspectral imaging. R- VCANet model is a combination of Rolling Guidance Filter and Vertex Component Analysis Network. R-VCANet is useful when there is limited sampling for feature extraction of hyperspectral data. R-VCANet is based on natural characteristics of HIS data, spectral properties, and spatial information. Hence the method proposed in [37] has performed better for hyperspectral image classification, especially when the sampling labels are limited.

Geophysics plays a vital role to study about graves detection after some changes are occurred in Buried graves. To detect the clandestine graves, local environment and different types of soils can be observed and data can be collected which is used for analysis. So it is important to become familiar with the equipment and hardware that is used for data acquisition and data analysis. John J. Schultz [38] used ground-penetrating radar for detection of clandestine graves. To use the GPR, it is also important to adjust the antenna for the best frequency of forensic work, which depends on spoidosol environment. Electromagnetic induction meter is also used to locate the clandestine graves, but EMI is limited for forensic research, so the lack of published research with the use of EMI meter to detect clandestinely buried bodies became deem in the forensic context. As mentioned above that EMI is limited for forensic research, a new idea is introduced to do better in the forensic field. According to that, buried remains can be easily detected by hyperspectral imaging. By hyperspectral image, a large scale of an image can be achieved and with the application of deep neural networks, it can be classified in a more précised way.

For un-mixing, classification and target detection of hyperspectral images, there are frequently used methods includes, sparse linear models, Gaussian mixture models, latent linear models, ensemble learning, directed graphical models, support vector machines, linear regression, logistic regression, Gaussian models, clustering and deep learning. The summary of all these methods is highlighted in Table II with referred research articles. 
TABLE II. METHODS FOR HYPERSPECTRAL IMAGING

\begin{tabular}{|c|c|c|c|c|c|c|}
\hline \multirow{2}{*}{ Methods } & \multicolumn{2}{|l|}{ Un-mixing } & \multicolumn{2}{|l|}{ Classification } & \multicolumn{2}{|c|}{ Target Detection } \\
\hline & Linear & Non-linear & Spatial-spectral & Pixel-wise & Target & Anomaly \\
\hline Sparse Linear Models & $\begin{array}{l}\text { spatial-spectral } \\
\text { [39] }\end{array}$ & & feature extraction [40] & feature extraction [41] & $\begin{array}{l}\text { spatial- } \\
\text { spectral } \\
{[39]}\end{array}$ & \\
\hline Gaussian Mixture Models & & & [42] & un-supervised [43] & & [44] \\
\hline Latent Linear Models & {$[45]$} & & feature extraction [46] & $\begin{array}{l}\text { dimensionality reduction } \\
\text { [47] }\end{array}$ & & \\
\hline Ensemble Learning & & & [48] & transfer learning [49] & & [50] \\
\hline Directed Graphical Models & $\begin{array}{l}\text { sub-pixel } \\
\text { mapping [51] }\end{array}$ & $\begin{array}{l}\text { spatial-spectral } \\
\text { [52] }\end{array}$ & & & & \\
\hline Support Vector Machines & $\begin{array}{l}\text { end member } \\
\text { extraction and } \\
\text { sub-pixel } \\
\text { mapping [53] }\end{array}$ & {$[54]$} & [55] & band selection [56] & [57] & [58] \\
\hline Linear Regression & [59] & {$[60]$} & & & & \\
\hline Logistic Regression & & & semi-supervised [61] & band selection [62] & & \\
\hline Gaussian Models & & & & transfer learning [63] & [64] & [65] \\
\hline Clustering & & & & un-supervised [66] & & [67] \\
\hline Deep Learning & & & $\begin{array}{l}\text { un-supervised feature } \\
\text { learning [68] } \\
\text { supervised feature } \\
\text { learning [69] }\end{array}$ & $\begin{array}{l}\text { supervised feature } \\
\text { learning [70] } \\
\text { un-supervised feature } \\
\text { learning [71] }\end{array}$ & & [72] \\
\hline
\end{tabular}

\section{FUTURE WORK}

To locate clandestine graves is a challenge for government (forensic department) after any victims. There is a lot of traditional ways to locate and detect the clandestine graves, for example with the help of forensic trained dogs, ground penetrating radar, electromagnetic induction meter, and hyper spectral sensor, in the forensic context. There are lots of researchers who are doing work to facilitate the detection of clandestine graves. In case of sudden disaster, the government forensic agencies play a vital role to detect the victims. Forensic archaeologist and anthropologists face a lot of challenges in forensic context.

One problem which Saudi emergency responders have to deal with is that sometimes heavy rains result in the flood. Victims of floods get buried deep in sand at ranges from $50 \mathrm{~cm}$ up to 2 meters. The soil under which the victims are buried is likely clayey loam and poorly drained, and it can be covered with water for some time (few days). The emergency responders focus their search operations in areas where victims are likely to be located such as valleys (e.g., Alhayer valley) shown in Fig. 4.

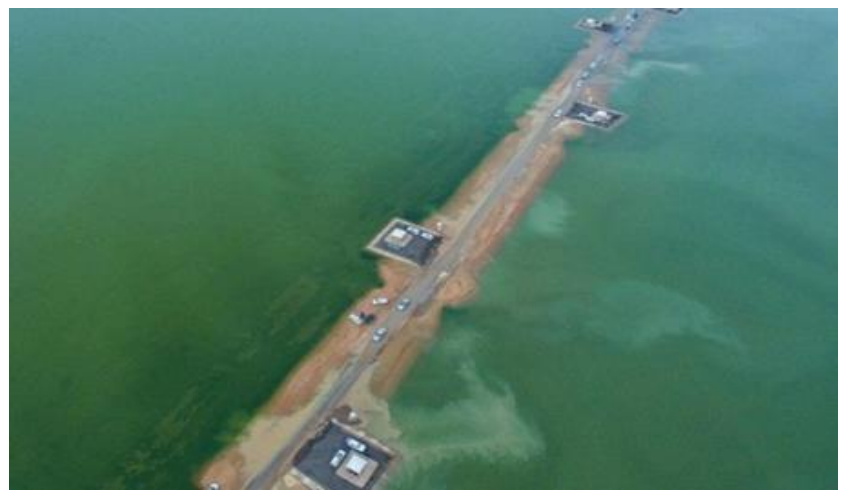

Fig. 4. Heavy Rain Flood in Alhayer Valley (Saudi Arabia).
However, the suspected areas are typically very large rendering conventional ways of grave detection such as using forensic detection dogs, ground-penetrating radars, and thermal imaging inefficient, let alone the huge cost and large human resources required in the search operations.

In this case, the idea of examining the emission and biogeochemistry of gases from graves and their detection through remote sensing, in particular, hyper-spectral images sounds like an interesting idea. A hyperspectral imager mounted on an Unmanned Airborne Vehicle (UAV) can fly over the suspected area and acquire imagery which may contain the absorption features (signature) of the gases of interest predominantly $\mathrm{CH} 4, \mathrm{~N} 2 \mathrm{O}$, and $\mathrm{CO} 2$. The higher pore air concentrations in graves and emission of methane, carbon dioxide and nitrous oxide to the atmosphere imply the existence of graves in that specific location, which can be associated with the missing victim. In contrast with dry sandy soil which is aerobic and therefore conducive to methane consumption and/or oxidation, the search area is clayey loam and poorly drained soil, and thus susceptible to methane production. This increases the chance of detecting gases of interest using hyper-spectral imaging techniques.

For better understanding about hyperspectral imaging using deep learning, we demonstrated the process of feature extraction using the deep neural network. In Fig. 5, there is a data patch of hyperspectral image for input layer and on the next number of hidden layers; hyperspectral image is further divided for feature extraction process. After the process of hybrid feature extraction results can be achieved from the output layer. The outcome can be in the form of 1 or 0 that shows the presence and absence of buried remains respectively. According to the current research trends, deep learning has become an advanced and robust technique to extract the features of hyperspectral image as compared to the traditional feature extraction techniques. 


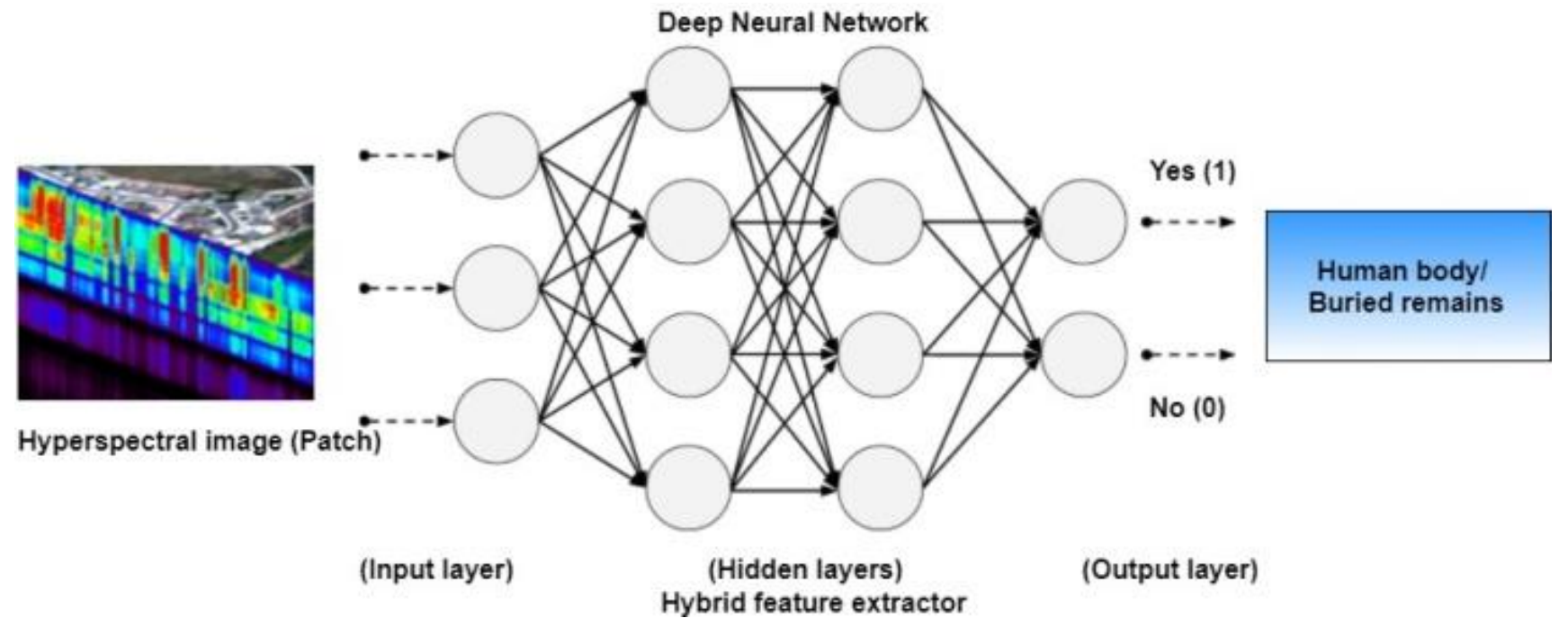

Fig. 5. Hyperspectral imaging using deep neural network.

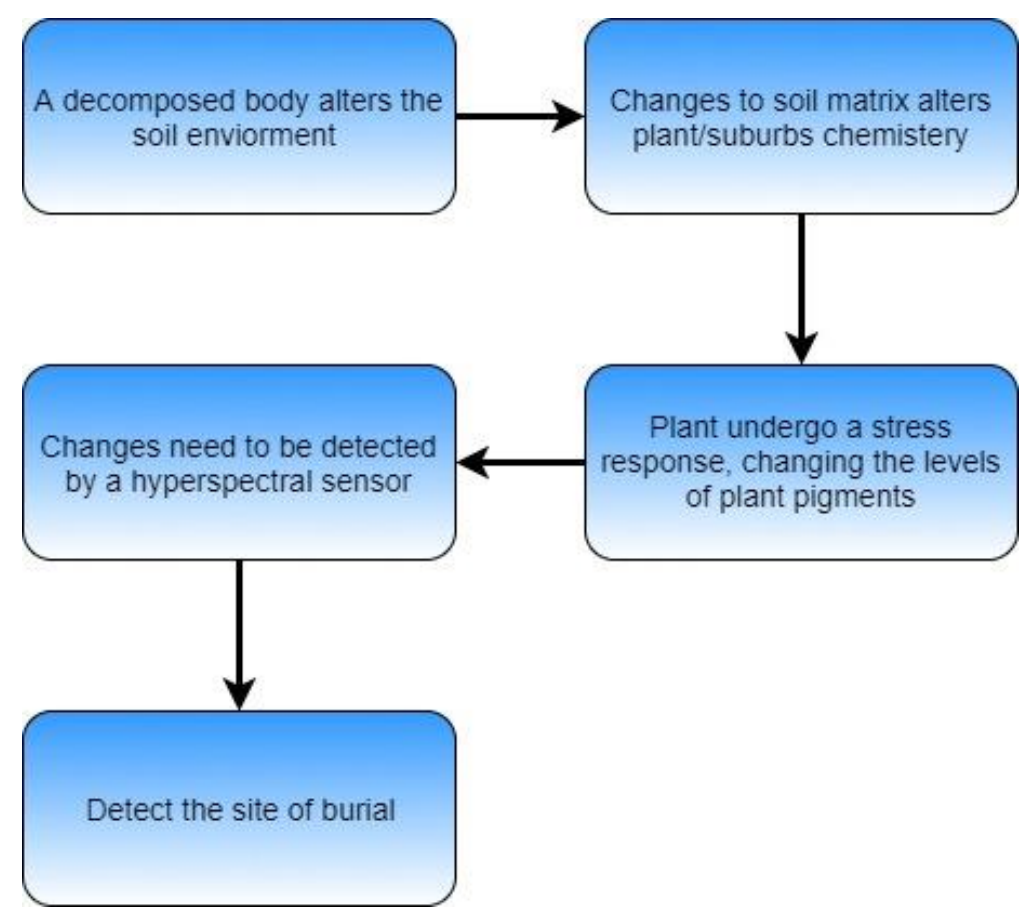

Fig. 6. Flowchart of future study.

The detection of clandestine and previously unknown burial sites is of interest to governments to start rescue operations. The detection of clandestine graves is an emerging tool in hyper-spectral remote sensing. In literature, studies have shown that it is possible to use hyperspectral remote sensing techniques in detection of mass graves. For this purpose, a UAV with hyperspectral sensors covering the visible to the shortwave infrared range was used to collect the imagery. The flow chart of the proposed technique is shown in Fig. 6 .

Hardware Required for Completion of Project:

- Hyperspectral Sensor (data acquisition)

- An Unmanned Airborne Vehicle (UAV)
- High spec PC/Laptop for experiment and data analysis Techniquesitools used for Completion of the Project:

- Matlab 2017

- Hybrid Feature Extraction

- Deep Neural Network (Classification)

\section{IMPLICATIONS OF HYPERSPECTRAL IMAGING}

Hyperspectral imaging is widely used in various applications such as Agriculture, Mineralogy, Surveillance, Physics, Astronomy, Chemical Imaging, and Environment. In the agricultural industry, diseases cause a serious loss for the economy. For suitable agriculture, it is important to monitor 
plants and trees for diseases. To detect diseases on early stage can save the plants and trees from further loss, Hyperspectral sensor can assist to control the virus by organized methods such as fungicide applications, disease-specific chemical applications and pesticide applications [73]. The hyperspectral imagery is used in land cover mapping. Land cover classification associated with the nature of land such as grassland, forest, concrete pavement, and sand etc. On the other hand, Land use indicates the human use of land, for example, industrial, residential and agricultural zone. Land use classification is an application used to classify the land surface such as Geometric correction, ground truth, and maximum likelihood. Land cover change detection is another application used to detect the changes in the earth surface. It can be identified with the comparison of existing image and the updated one. Changes on earth have two main types. One is seasonal change and other is annual change, in seasonal change forests or plants get changes according to the season, but in annual changes, new things take place for example developments on the earth or deforested place. Global vegetation map is another application of hyperspectral imaging. According to this application global vegetation index data contains information of normalized difference vegetation index (NDVI). NDVI is updated on the weekly basis, so it can also contain some noisy data. Water is an essential part of life. Water quality monitoring is an important application of hyperspectral imager. Quality of water can be analyzed due to its greenish or yellowish shaded color. To measure the surface temperature of sea hyperspectral imager can also perform the services. The hyperspectral sensor can also provide thermal information for a short interval of time over a large amount of area shown in Fig. 7. Brightness Temperature is a sensor used to detect the temperature of objects.

Every object has different emissivity which discharges electromagnetic energy. The value of emissivity is nearly equal to 1 and remains constant as compared with the temperature of the earth. Snow covered area is also the detectable area for hyperspectral sensors. During snow survey, snow water equivalent has been planned with the approximation of snow-covered area. Height measurement can also be calculated by Hyperspectral imager with the matchup of stereo images. In [74], there are two techniques to measure the height of objects, one is already defined as stereo matching and other is based on analytical plotters.

Fruits are the important part of food because it provides vitamins and energy to human's body. To gain healthy and fresh fruits is also a challenge. Hyperspectral imager provides the applications to monitor the quality of fruits. Peng and Lu [75] developed a reflective system to identify apple firmness and solids contents with the use of steady object stage. With the help of optical fiber and focusing lenses, 2-dimensional hyperspectral images were obtained. Huang and Lu [76] introduced another quality attribute analyzed by hyperspectral imaging is known as mealiness. Haung and Lu analyzed the association between apple mealiness and reflective hyperspectral line images. The mealiness of apple was calculated by the solidity and ripeness.

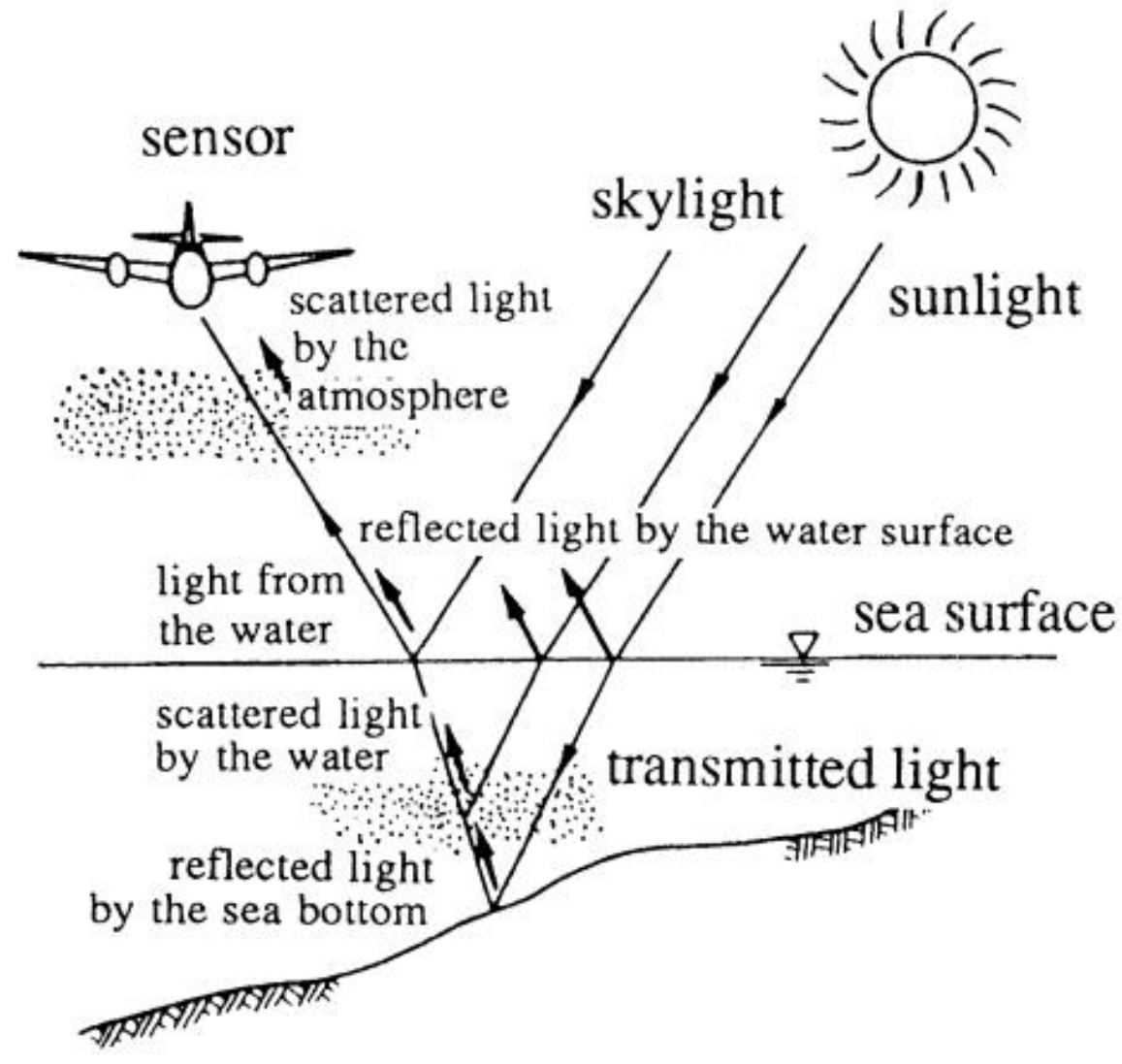

Fig. 7. Incident light into a sensor on the sea. 
Remote sensing of vegetation is widely used in the world to check the quality of vegetables. The important application of hyperspectral imaging about vegetables including mushrooms, onions, cherry tomato, and spinach leaves. Ariana and Lu [77] designed a VIS-NIR hyperspectral imaging system joining reflective mode and transmitted mode collectively, while with the use of moving transport proposal. The system was able to identify internal issues of pickles and cucumbers which were impossible to visualize by the human eye. Hyperspectral sensors can also be used to monitor the quality of meat such as pork, chicken, and beef. In the context of pattern recognition, marbling is used to detect the quality of meat. To extract the marbling, a proposed technique used a wide line detector implemented by pattern recognition [78]. Results are achieved with the help of standard marbling charts and classified with the accuracy of 99 per cent. Hyperspectral imaging is also applied for seafood such as prawn, cod and smoked salmon. Near Infrared (NIR) Hyperspectral System was designed to detect the grains diseases on the early stage with the use of mathematical modeling framework. This system was based on supervised classification [79]. In [80], Khan et al. studied on advanced developments in the field of HIS Analysis. Details of this review based on fundamentals of Hyperspectral Imaging including spatial resolution, spectral resolution, temporal resolution, feature extraction. Secondly, authors explained the advance applications of hyperspectral imaging such as food quality, safety, security, remote sensing, especially in the field of forensic documents examination to study about intrinsic and extrinsic elements. After a long discussion about hyperspectral imaging, we have come to know that hyperspectral imaging is an active area for research, through which new ideas can be implemented and the world can be changed.

\section{CONCLUSION}

In this paper, we described the fundamental concept, hyperspectral remote sensing, remotely sensed information, methods for hyperspectral imaging and applications based on hyperspectral imaging. We introduced a better approach to detect the buried remains with the use of deep neural networks for feature extraction. Finally, we focused on the use of hyperspectral imaging in different applications. However, hyperspectral imaging technology using deep learning is gradually becoming a great opportunity for researchers, in the field of biomedical, vegetation, especially in the forensic context.

\section{ACKNOWLEDGMENT}

This research was supported by the Basic and Advanced Research Projects in Chongqing, China under Grant No.61672117.

\section{REFERENCES}

[1] Ahmadi, S. B. B., Nanehkaran, Y. A. and Layazali, S. Review on hyperspectral imaging system. Int. J. Sci. Eng. Res., 4, 5 2013), 253-258.

[2] Tong, Q., Zhang, B. and Zheng, L. S. Hyperspectral remote sensing technology and applications in China2004).

[3] Feng, Y.-Z. and Sun, D.-W. Application of hyperspectral imaging in food safety inspection and control: a review. Critical reviews in food science and nutrition, 52, 11 2012), 1039-1058.
[4] Wang, H., Peng, J., Xie, C., Bao, Y. and He, Y. Fruit quality evaluation using spectroscopy technology: a review. Sensors, 15, 5 2015), 1188911927.

[5] Bonnier, F., Bertrand, D., Rubin, S., Venteo, L., Pluot, M., Baehrel, B., Manfait, M. and Sockalingum, G. Detection of pathological aortic tissues by infrared multispectral imaging and chemometrics. Analyst, 133, 6 2008), 784-790.

[6] Lanaras, C., Baltsavias, E. and Schindler, K. ADVANCES IN HYPERSPECTRAL AND MULTISPECTRAL IMAGE FUSION AND SPECTRAL UNMIXING. International Archives of the Photogrammetry, Remote Sensing \& Spatial Information Sciences, 402015).

[7] Sun, L., Wu, Z., Liu, J., Xiao, L. and Wei, Z. Supervised spectral-spatial hyperspectral image classification with weighted Markov random fields. IEEE Transactions on Geoscience and Remote Sensing, 53, 3 2015), 1490-1503.

[8] Sun, L., Jeon, B., Zheng, Y. and Wu, Z. Hyperspectral image restoration using low-rank representation on spectral difference image. IEEE Geoscience and Remote Sensing Letters, 14, 7 2017), 1151-1155.

[9] Wu, Z., Shi, L., Li, J., Wang, Q., Sun, L., Wei, Z., Plaza, J. and Plaza, A. GPU parallel implementation of spatially adaptive hyperspectral image classification. IEEE Journal of Selected Topics in Applied Earth Observations and Remote Sensing, 11, 4 2018), 1131-1143.

[10] Sun, L., Jeon, B., Soomro, B. N., Zheng, Y., Wu, Z. and Xiao, L. Fast Superpixel Based Subspace Low Rank Learning Method for Hyperspectral Denoising. IEEE Access, 62018), 12031-12043.

[11] Goetz, A. F., Vane, G., Solomon, J. E. and Rock, B. N. Imaging spectrometry for earth remote sensing. Science, 228, 4704 1985), 11471153.

[12] Moser, G., Serpico, S. B. and Benediktsson, J. A. Land-cover mapping by Markov modeling of spatial-contextual information in very-highresolution remote sensing images. Proceedings of the IEEE, 101, 3 2013), 631-651.

[13] Bioucas-Dias, J. M., Plaza, A., Camps-Valls, G., Scheunders, P., Nasrabadi, N. and Chanussot, J. Hyperspectral remote sensing data analysis and future challenges. IEEE Geoscience and remote sensing magazine, 1, 2 2013), 6-36.

[14] Brook, A. and Ben-Dor, E. Advantages of the boresight effect in hyperspectral data analysis. Remote Sensing, 3, 3 2011), 484-502.

[15] Mutanga, O. and Skidmore, A. K. Continuum-removed absorption features estimate tropical savanna grass quality in situ. City, 2003.

[16] Díaz-Delgado, R. and Pons, X. Spatial patterns of forest fires in Catalonia (NE of Spain) along the period 1975-1995: analysis of vegetation recovery after fire. Forest ecology and management, 147, 1 2001), 67-74.

[17] Mutanga, O., Van Aardt, J. and Kumar, L. Imaging spectroscopy (hyperspectral remote sensing) in southern Africa: an overview. South African Journal of Science, 105, 5-6 2009), 193-198.

[18] Walter, V. Object-based classification of remote sensing data for change detection. ISPRS Journal of photogrammetry and remote sensing, 58, 34 2004), 225-238.

[19] Roujean, J. L., Leroy, M. and Deschamps, P. Y. A bidirectional reflectance model of the Earth's surface for the correction of remote sensing data. Journal of Geophysical Research: Atmospheres, 97, D18 1992), 20455-20468.

[20] Benz, U. C., Hofmann, P., Willhauck, G., Lingenfelder, I. and Heynen, M. Multi-resolution, object-oriented fuzzy analysis of remote sensing data for GIS-ready information. ISPRS Journal of photogrammetry and remote sensing, 58, 3-4 2004), 239-258.

[21] Liao, D., Qian, Y., Zhou, J. and Tang, Y. Y. A manifold alignment approach for hyperspectral image visualization with natural color. IEEE Transactions on Geoscience and Remote Sensing, 54, 6 2016), 31513162.

[22] Park, B., Yoon, S.-C., Windham, W. R., Lawrence, K. C., Kim, M. S. and Chao, K. Line-scan hyperspectral imaging for real-time in-line poultry fecal detection. Sensing and instrumentation for food quality and safety, 5, 1 2011), 25-32. 
[23] Burns, D. A. and Ciurczak, E. W. Handbook of near-infrared analysis. CRC press, 2007.

[24] Cen, H. and He, Y. Theory and application of near infrared reflectance spectroscopy in determination of food quality. Trends in Food Science \& Technology, 18, 2 2007), 72-83.

[25] Yoon, J. H., Sheremata, S., Rokem, A. and Silver, M. A. Windows to the soul: vision science as a tool for studying biological mechanisms of information processing deficits in schizophrenia. Frontiers in psychology, 42013), 681.

[26] Qian, Y., Yao, F. and Jia, S. Band selection for hyperspectral imagery using affinity propagation. IET Computer Vision, 3, 4 2009), 213-222.

[27] Jia, S., Ji, Z., Qian, Y. and Shen, L. Unsupervised band selection for hyperspectral imagery classification without manual band removal. IEEE Journal of Selected Topics in Applied Earth Observations and Remote Sensing, 5, 2 2012), 531-543.

[28] Qian, Y. and Ye, M. Hyperspectral imagery restoration using nonlocal spectral-spatial structured sparse representation with noise estimation. IEEE Journal of Selected Topics in Applied Earth Observations and Remote Sensing, 6, 2 2013), 499-515.

[29] Naganathan, G. K., Grimes, L. M., Subbiah, J., Calkins, C. R., Samal, A. and Meyer, G. E. Visible/near-infrared hyperspectral imaging for beef tenderness prediction. Computers and electronics in agriculture, 64, 2 2008), 225-233.

[30] Kim, I., Kim, M., Chen, Y. and Kong, S. Detection of skin tumors on chicken carcasses using hyperspectral fluorescence imaging. Transactions of the ASAE, 47, 5 2004), 1785.

[31] Liu, L. and Ngadi, M. Detecting fertility and early embryo development of chicken eggs using near-infrared hyperspectral imaging. Food and Bioprocess Technology, 6, 9 2013), 2503-2513.

[32] Lu, D. and Weng, Q. A survey of image classification methods and techniques for improving classification performance. International journal of Remote sensing, 28, 5 2007), 823-870.

[33] Friedl, M., Woodcock, C., Gopal, S., Muchoney, D., Strahler, A. and Barker-Schaaf, C. A note on procedures used for accuracy assessment in land cover maps derived from AVHRR data2000).

[34] Liang, J., Zhou, J., Qian, Y., Wen, L., Bai, X. and Gao, Y. On the sampling strategy for evaluation of spectral-spatial methods in hyperspectral image classification. IEEE Transactions on Geoscience and Remote Sensing, 55, 2 2017), 862-880.

[35] Mas, J.-F., Pérez-Vega, A., Ghilardi, A., Martínez, S., Loya-Carrillo, J. O. and Vega, E. A suite of tools for assessing thematic map accuracy. Geography Journal, 20142014).

[36] Petersson, H., Gustafsson, D. and Bergstrom, D. Hyperspectral image analysis using deep learning - a review. IEEE, City, 2016.

[37] Pan, B., Shi, Z. and Xu, X. R-VCANet: a new deep-learning-based hyperspectral image classification method. IEEE Journal of Selected Topics in Applied Earth Observations and Remote Sensing, 10, 5 2017), 1975-1986.

[38] Roedl, G., Elmes, G. A. and Conley, J. Spatial technology applications. Springer, City, 2014.

[39] Iordache, M.-D., Bioucas-Dias, J. M. and Plaza, A. Total variation spatial regularization for sparse hyperspectral unmixing. IEEE Transactions on Geoscience and Remote Sensing, 50, 11 2012), 44844502.

[40] Du, P., Xue, Z., Li, J. and Plaza, A. Learning discriminative sparse representations for hyperspectral image classification. IEEE Journal of Selected Topics in Signal Processing, 9, 6 2015), 1089-1104.

[41] Charles, A. S., Olshausen, B. A. and Rozell, C. J. Learning sparse codes for hyperspectral imagery. IEEE Journal of Selected Topics in Signal Processing, 5, 5 2011), 963.

[42] Yang, H., Du, Q. and Ma, B. Decision fusion on supervised and unsupervised classifiers for hyperspectral imagery. IEEE Geoscience and Remote Sensing Letters, 7, 4 2010), 875-879.

[43] Tarabalka, Y., Benediktsson, J. A. and Chanussot, J. Spectral-spatial classification of hyperspectral imagery based on partitional clustering techniques. IEEE Transactions on Geoscience and Remote Sensing, 47, 8 2009), 2973-2987.
[44] Tarabalka, Y., Haavardsholm, T. V., Kåsen, I. and Skauli, T. Real-time anomaly detection in hyperspectral images using multivariate normal mixture models and GPU processing. Journal of Real-Time Image Processing, 4, 3 2009), 287-300.

[45] Nascimento, J. M. and Dias, J. M. Does independent component analysis play a role in unmixing hyperspectral data? IEEE Transactions on Geoscience and Remote Sensing, 43, 12005$),$ 175-187.

[46] Plaza, J., Plaza, A. J. and Barra, C. Multi-channel morphological profiles for classification of hyperspectral images using support vector machines. Sensors, 9, 1 2009), 196-218.

[47] Shaw, G. and Manolakis, D. Signal processing for hyperspectral image exploitation. IEEE Signal processing magazine, 19, 1 2002), 12-16.

[48] Merentitis, A., Debes, C. and Heremans, R. Ensemble learning in hyperspectral image classification: Toward selecting a favorable biasvariance tradeoff. IEEE Journal of Selected Topics in Applied Earth Observations and Remote Sensing, 7, 4 2014), 1089-1102.

[49] Rajan, S., Ghosh, J. and Crawford, M. M. Exploiting class hierarchies for knowledge transfer in hyperspectral data. IEEE Transactions on Geoscience and Remote Sensing, 44, 11 2006), 3408-3417.

[50] Peerbhay, K. Y., Mutanga, O. and Ismail, R. Random forests unsupervised classification: The detection and mapping of solanummauritianum infestations in plantation forestry using hyperspectral data. IEEE Journal of Selected Topics in Applied Earth Observations and Remote Sensing, 8, 6 2015), 3107-3122.

[51] Zhao, J., Zhong, Y., Wu, Y., Zhang, L. and Shu, H. Sub-pixel mapping based on conditional random fields for hyperspectral remote sensing imagery. IEEE Journal of Selected Topics in Signal Processing, 9, 6 2015), 1049-1060.

[52] Altmann, Y., Pereyra, M. and McLaughlin, S. Bayesian nonlinear hyperspectral unmixing with spatial residual component analysis. IEEE Transactions on Computational Imaging, 1, 3 2015), 174-185.

[53] Villa, A., Chanussot, J., Benediktsson, J. A. and Jutten, C. Spectral unmixing for the classification of hyperspectral images at a finer spatial resolution. IEEE Journal of Selected Topics in Signal Processing, 5, 3 2011), 521-533.

[54] Gu, Y., Wang, S. and Jia, X. Spectral unmixing in multiple-kernel hilbert space for hyperspectral imagery. IEEE Transactions on Geoscience and Remote Sensing, 51, 7 2013), 3968-3981.

[55] Benediktsson, J. A., Palmason, J. A. and Sveinsson, J. R. Classification of hyperspectral data from urban areas based on extended morphological profiles. IEEE Transactions on Geoscience and Remote Sensing, 43, 3 2005), 480-491.

[56] Bazi, Y. and Melgani, F. Toward an optimal SVM classification system for hyperspectral remote sensing images. IEEE Transactions on Geoscience and Remote Sensing, 44, 11 2006), 3374-3385.

[57] Sakla, W., Chan, A., Ji, J. and Sakla, A. An SVDD-based algorithm for target detection in hyperspectral imagery. IEEE Geoscience and Remote Sensing Letters, 8, 2 2011), 384-388.

[58] Gurram, P. and Kwon, H. Support-vector-based hyperspectral anomaly detection using optimized kernel parameters. IEEE Geoscience and Remote Sensing Letters, 8, 6 2011), 1060-1064.

[59] Heinz, D. C. Fully constrained least squares linear spectral mixture analysis method for material quantification in hyperspectral imagery. IEEE Transactions on Geoscience and Remote Sensing, 39, 3 2001), 529-545.

[60] Heylen, R., Scheunders, P., Rangarajan, A. and Gader, P. Nonlinear unmixing by using different metrics in a linear unmixing chain. IEEE Journal of Selected Topics in Applied Earth Observations and Remote Sensing, 8, 6 2015), 2655-2664.

[61] Dópido, I., Li, J., Gamba, P. and Plaza, A. A new hybrid strategy combining semisupervised classification and unmixing of hyperspectral data. IEEE Journal of Selected Topics in Applied Earth Observations and Remote Sensing, 7, 8 2014), 3619-3629.

[62] Cheng, Q., Varshney, P. K. and Arora, M. K. Logistic regression for feature selection and soft classification of remote sensing data. IEEE Geoscience and Remote Sensing Letters, 3, 4 2006), 491-494.

[63] Persello, C. and Bruzzone, L. Active learning for domain adaptation in the supervised classification of remote sensing images. IEEE 
Transactions on Geoscience and Remote Sensing, 50, 11 2012), 44684483.

[64] Manolakis, D., Marden, D. and Shaw, G. A. Hyperspectral image processing for automatic target detection applications. Lincoln laboratory journal, 14, 1 2003), 79-116.

[65] Chang, C.-I. and Chiang, S.-S. Anomaly detection and classification for hyperspectral imagery. IEEE Transactions on Geoscience and Remote Sensing, 40, 6 2002), 1314-1325.

[66] Villa, A., Chanussot, J., Benediktsson, J. A., Jutten, C. and Dambreville, R. Unsupervised methods for the classification of hyperspectral images with low spatial resolution. Pattern Recognition, 46, 6 2013), 15561568.

[67] Balas, C., Epitropou, G. and Pappas, C. Multi/hyper-spectral imaging. Handbook of Biomedical Optics2011), 131-164.

[68] Zhao, W., Guo, Z., Yue, J., Zhang, X. and Luo, L. On combining multiscale deep learning features for the classification of hyperspectral remote sensing imagery. International journal of Remote sensing, 36, 13 2015), 3368-3379.

[69] Hu, W., Huang, Y., Wei, L., Zhang, F. and Li, H. Deep convolutional neural networks for hyperspectral image classification. Journal of Sensors, 20152015).

[70] Zhu, X. X., Tuia, D., Mou, L., Xia, G.-S., Zhang, L., Xu, F. and Fraundorfer, F. Deep learning in remote sensing: a comprehensive review and list of resources. IEEE Geoscience and remote sensing magazine, 5, 4 2017), 8-36.

[71] Chen, Y., Zhao, X. and Jia, X. Spectral-spatial classification of hyperspectral data based on deep belief network. IEEE Journal of Selected Topics in Applied Earth Observations and Remote Sensing, 8, 6 2015), 2381-2392.
[72] Li, W., Wu, G. and Du, Q. Transferred Deep Learning for Anomaly Detection in Hyperspectral Imagery. IEEE Geosci. Remote Sensing Lett., 14, 5 2017), 597-601.

[73] Sankaran, S., Mishra, A., Ehsani, R. and Davis, C. A review of advanced techniques for detecting plant diseases. Computers and electronics in agriculture, 72, 1 2010), 1-13.

[74] Duvvuru, R., Rao, G. N., Bendalam, S., Gemechu, R., Chundi, A., Naidu, D. S., Rao, C. R. and Rao, P. J. A Case Study On Child LabourGIS Approach.

[75] Mendoza, F., Lu, R., Ariana, D., Cen, H. and Bailey, B. Integrated spectral and image analysis of hyperspectral scattering data for prediction of apple fruit firmness and soluble solids content. Postharvest Biology and Technology, 62, 2 2011), 149-160.

[76] Huang, M. and Lu, R. Apple mealiness detection using hyperspectral scattering technique. Postharvest Biology and Technology, 58, 3 2010), 168-175.

[77] Ariana, D. P. and Lu, R. Evaluation of internal defect and surface color of whole pickles using hyperspectral imaging. Journal of Food Engineering, 96, 4 2010), 583-590.

[78] Liu, L., Ngadi, M., Prasher, S. and Gariépy, C. Objective determination of pork marbling scores using the wide line detector. Journal of Food Engineering, 110, 3 2012), 497-504.

[79] Arngren, M., Hansen, P. W., Eriksen, B., Larsen, J. and Larsen, R. Analysis of pregerminated barley using hyperspectral image analysis. Journal of agricultural and food chemistry, 59, 21 2011), 11385-11394.

[80] Khan, M. J., Khan, H. S., Yousaf, A., Khurshid, K. and Abbas, A. Modern trends in hyperspectral image analysis: a review. IEEE Access, 62018), 14118-14129. 